
abr-jun 2005.

\title{
Envelhecimento acelerado em sementes de pepino com e sem solução salina saturada
}

\author{
Salvador Barros Torres \\ EMPARN, C. Postal 188, 59062-500 Natal-RN; Bolsista CNPq; E-mail: sbtorres@digizap.com.br
}

\begin{abstract}
RESUMO
Estudou-se a metodologia do teste de envelhecimento acelerado na obtenção do potencial fisiológico de sementes de pepino (Cucumis sativus L.), incluindo a avaliação da eficiência do uso de solução saturada de sal no controle da absorção de água pelas sementes. Cinco lotes de sementes, cultivar Rubi, foram submetidos aos testes de germinação, emergência de plântulas em casa de vegetação e envelhecimento acelerado (períodos de 48,72 ou 96 horas, a $38^{\circ} \mathrm{C}$ ou $41^{\circ} \mathrm{C}$ ), com e sem o uso de solução saturada de $\mathrm{NaCl}$. O teste de envelhecimento acelerado (procedimento convencional e com solução salina), conduzido com períodos de exposição de 72 e 96 h a 38 ou $41^{\circ} \mathrm{C}$, apresenta sensibilidade suficiente para a avaliação do potencial fisiológico de sementes de pepino. A utilização de solução saturada de $\mathrm{NaCl}$ contribui para reduzir a absorção de água pelas sementes de pepino durante o teste de envelhecimento acelerado, acarreta taxa de deterioração menos acentuada, possibilitando a obtenção de resultados menos drásticos e mais uniformes, sem afetar a eficiência do teste.
\end{abstract}

Palavras-chave: Cucumis sativus, potencial fisiológico, vigor.

\begin{abstract}
Accelerated aging of cucumber seeds submitted to saturated salt solution

Different procedures of the accelerated aging test were evaluated to obtain the physiological quality of cucumber (Cucumis sativus L.) seeds and the efficiency of using saturated salt solution in the control of water uptake by seeds. Five seed lots of the cv Rubi, were tested for germination, seedling emergence, conventional accelerated aging and salt saturated accelerated aging tests. Results showed that the accelerated aging test (conventional and salt saturated), using the 72 and $96 \mathrm{~h}$, at 38 or $41^{\circ} \mathrm{C}$ combinations was sufficiently sensitive to detect differences in physiological quality of cucumber seeds. We observed that seed water content after the salt saturated accelerated aging was lower and more uniform, thus presenting advantages in relation to the conventional procedure.
\end{abstract}

Keywords: Cucumis sativus, physiological potential, vigor.

(Recebido para publicação em 20 de maio de 2004 e aceito em 26 de janeiro de 2005)

$\mathrm{O}$ pepino (Cucumis sativus L.) é uma hortaliça de grande importância no Brasil, sendo consumido como salada ou conserva. Em 2002, a CEAGESP (AGRIANUAL, 2004) comercializou 22.025 toneladas o que o destaca como uma olerícola de grande valor comercial. As sementes, por apresentarem alto valor comercial, merecem atenção especial quanto ao potencial fisiológico. Entretanto, para esta espécie, verifica-se a quase inexistência de pesquisas direcionadas à comparação de testes de vigor, sobretudo em nossas condições.

Para algumas culturas, testes específicos têm mostrado eficiência, como o de frio para sementes de milho (Zea mays L.), o de envelhecimento acelerado para as de soja [Glycine max (L.) Merrill] e o de condutividade elétrica para as de ervilha (Pisum sativum L.). Por outro lado, poucos estudos têm sido conduzidos sobre o uso dos testes de vigor para avaliação do potencial fisiológico de sementes de hortaliças, com exceção do teste de deterioração controlada, desenvolvido por Powell e Matthews (1981).
O teste de envelhecimento acelerado é reconhecido como um dos mais utilizados para a avaliação do potencial fisiológico de sementes de várias espécies, proporcionando informações com alto grau de consistência (TEKRONY, 1995). O princípio está baseado na aceleração artificial da taxa de deterioração das sementes, pela sua exposição a níveis elevados de temperatura e umidade relativa do ar, considerados os fatores ambientais preponderantes na intensidade e velocidade de deterioração (MARCOS-FILHO, 1999b). Nessa situação, sementes de baixa qualidade deterioram-se mais rapidamente do que as mais vigorosas, apresentando redução diferenciada da viabilidade.

Vários fatores afetam o comportamento das sementes submetidas ao teste; a interação temperatura/período de exposição é um dos mais estudados. Alguns autores se dedicaram ao estudo dessa interação, indicando, para sementes de cebola, $41^{\circ} \mathrm{C} / 72 \mathrm{~h}$ (TeKrony, 1995); pimentão, $41^{\circ} \mathrm{C} / 72 \mathrm{~h}$
(PANOBIANCO; MARCOS-FILHO, 1998); brócolis, $45^{\circ} \mathrm{C} / 48 \mathrm{~h}$ (TEBALDI et al., 1999); pepino, $41^{\circ} \mathrm{C} / 48$ e $72 \mathrm{~h}$ (BHERING et al., 2000); tomate, $41^{\circ} \mathrm{C} /$ $72 \mathrm{~h}$ (PANOBIANCO; MARCOS-FILHO, 2001) e melão, $38^{\circ} \mathrm{C}$ ou $41^{\circ} \mathrm{C} / 72$ e $96 \mathrm{~h}$ (Torres e MARCOS-FILHO, 2003).

Outro aspecto a ser considerado no teste de envelhecimento acelerado, são as diferenças na absorção de água pelas sementes que, expostas a atmosfera úmida, podem apresentar variações acentuadas no grau de umidade. Pesquisas conduzidas com espécies de sementes pequenas têm revelado resultados pouco consistentes devido à variação muito acentuada do grau de umidade das amostras, após o envelhecimento (POWELL, 1995). Nesse sentido, vêm sendo estudadas alternativas para a condução do envelhecimento acelerado com sementes dessas espécies, com a substituição da água por soluções saturadas de sais. Dependendo da solução utilizada, são obtidos níveis específicos de umidade relativa do ar, permitindo re- 
duzir a taxa de absorção de água, a velocidade e a intensidade de deterioração das sementes (JIANHUA; MCDONALD, 1997), sem reduzir a sensibilidade do teste. Alguns autores constataram maior eficiência do teste de envelhecimento acelerado com o uso de soluções saturadas de sal na classificação dos lotes; entre eles, Panobianco e Marcos-Filho (1998), com sementes de pimentão; Rodo et al. (2000), com cenoura, Bennett et al. (2001), com milho doce e Torres e Marcos-Filho (2003), com melão.

O presente trabalho teve por objetivo estudar a metodologia do teste de envelhecimento acelerado para a avaliação do potencial fisiológico de sementes de pepino, estabelecendo comparação entre o procedimento convencional e a utilização de solução saturada de cloreto de sódio.

\section{MATERIAL E MÉTODOS}

O trabalho foi conduzido em laboratório da EMPARN, em Natal (RN). Para isso, foram utilizados cinco lotes de sementes de pepino, cv. Rubi, adquiridos de cinco produtores do Rio Grande do Norte. Após a recepção, as sementes foram homogeneizadas em divisor tipo solo, e acondicionadas em saco de papel Kraft e armazenadas em condições controladas $\left(18-20^{\circ} \mathrm{C}\right.$ e $60 \%$ de umidade relativa do ar), permanecendo nessas condições até o final da fase experimental. As avaliações da qualidade das sementes foram realizadas por meio dos seguintes testes: grau de umidade, realizado em estufa a $105 \pm 3^{\circ} \mathrm{C} / 24 \mathrm{~h}$, utilizando-se quatro subamostras com cerca de $5 \mathrm{~g}$ para cada lote (BRASIL, 1992); germinação, utilizando-se quatro subamostras de 50 sementes por lote, distribuídas em rolos de papel toalha Germitest, umedecidas com quantidade de água equivalente a 2,5 vezes o peso do substrato seco e colocadas para germinar a $25^{\circ} \mathrm{C}$. As avaliações foram realizadas aos quatro e oito dias após a semeadura, segundo os critérios estabelecidos pelas Regras para Análise de Sementes (BRASIL, 1992); os resultados foram expressos em porcentagem média de plântulas normais, para cada lote; emergência de plântulas em casa de vegetação, utilizando-se quatro subamostras de 50 sementes por lote, semeadas individualmente em bandejas multicelulares de "isopor" com células

Tabela 1. Grau de umidade (\%), germinação (\%) e emergência de plântulas em casa de vegetação (\%) de sementes de cinco lotes de pepino, cultivar Rubi. Natal, EMPARN, 2004.

\begin{tabular}{cccc}
\hline Lote & $\begin{array}{c}\text { Grau de umidade } \\
(\%)\end{array}$ & Germinação (\%) & $\begin{array}{c}\text { Emergência de } \\
\text { plântulas (\%) }\end{array}$ \\
\hline 1 & 7,1 & $92 \mathrm{a}$ & $88 \mathrm{~b}$ \\
2 & 7,4 & $94 \mathrm{a}$ & $92 \mathrm{a}$ \\
3 & 7,2 & $96 \mathrm{a}$ & $90 \mathrm{a}$ \\
4 & 7,2 & $98 \mathrm{a}$ & $92 \mathrm{a}$ \\
5 & 7,3 & $98 \mathrm{a}$ & $92 \mathrm{a}$ \\
\hline $\mathrm{CV}(\%)$ & - & 2,4 & 4,2 \\
\hline
\end{tabular}

*Na coluna, valores seguidos pela mesma letra não diferem entre si pelo teste de Tukey a 5\%.

separadas, contendo substrato comercial (Plantimax - Hortaliças). As bandejas foram mantidas entre $25^{\circ} \mathrm{C}$ e $30^{\circ} \mathrm{C}$, em casa de vegetação dotada de sistema de nebulização intermitente. As avaliações foram realizadas aos 21 dias após a semeadura, determinando-se a porcentagem de emergência de plântulas por lote; envelhecimento acelerado (procedimento convencional), utilizanod-se caixas tipo "gerbox", como compartimento individual (mini-câmara), possuindo em seu interior uma bandeja com tela de alumínio, onde as sementes foram distribuídas de maneira a formarem camada uniforme. Dentro de cada compartimento individual foram adicionados $40 \mathrm{ml}$ de água destilada. As caixas, tampadas; foram mantidas em incubadora por três períodos de envelhecimento $(48,72$ e 96 horas), sendo utilizadas duas temperaturas $\left(38^{\circ} \mathrm{C} \mathrm{e} 41^{\circ} \mathrm{C}\right)$. Decorrido esse período de envelhecimento, quatro subamostras de 50 sementes por tratamento foram colocadas para germinar conforme metodologia descrita para o teste de germinação. A avaliação foi realizada aos oito após a semeadura e os resultados expressos em porcentagem média de plântulas normais para cada lote. Para fins de monitoramento do teste, foi determinado, também, o grau de umidade das sementes antes e após os períodos de envelhecimento; envelhecimento acelerado (solução saturada de sal), conduzido de maneira similar a descrita para o teste convencional, com exceção de serem adicionados ao fundo de cada caixa plástica (compartimento individual), $40 \mathrm{ml}$ de solução saturada de $\mathrm{NaCl}$, em substituição à água. Essa solução foi obtida pela proporção $40 \mathrm{~g}$ de $\mathrm{NaCl} /$ $100 \mathrm{ml}$ de água, estabelecendo, com isso, ambiente com umidade relativa do ar de $76 \%$, conforme procedimento proposto por Jianhua e McDonald (1997).

Utilizou-se o delineamento estatístico inteiramente casualizado, com qua- tro repetições verdadeiras e as análises realizadas separadamente para cada teste. Os dados dos testes de germinação, emergência e envelhecimento acelerado foram previamente transformados em arc sen $\sqrt{x / 100}$ mas os valores originais foram empregados nas Tabelas. A comparação das médias foi feita pelo teste de Tukey, a 5\% de probabilidade.

\section{RESULTADOS E DISCUSSÃO}

Os dados referentes ao grau de umidade das sementes foram semelhantes para os cinco lotes (Tabela 1). Este fato é importante para a execução das avaliações de envelhecimento acelerado, considerando-se que a uniformização do teor de água das sementes é imprescindível para a padronização das avaliações e obtenção de resultados consistentes (MARCOS-FILHO, 1999a), pois, dentro de certos limites, as sementes mais úmidas são mais afetadas pelas condições do envelhecimento acelerado.

Pelo teste de germinação (Tabela 1), verificou-se que não houve diferenciação estatística entre os lotes. Segundo Marcos-Filho (1999a), é importante e coerente a comparação de lotes de sementes com germinação semelhante e, de acordo com Powell (1981), preferencialmente situados na Fase I da curva de perda de viabilidade, pois, ao atingir a Fase II, mesmo o teste germinação (conduzido sob condições favoráveis) é capaz de detectar diferenças no potencial fisiológico das amostras avaliadas. Essa autora considera que a posição de cada lote dentro da Fase I determina seu nível de vigor. No presente estudo, todos os lotes apresentaram germinação variando entre $92 \%$ e $98 \%$, estando situados, portanto, na Fase I da curva de 
Tabela 2. Dados médios de plântulas normais (\%) obtidos para os testes de envelhecimento acelerado convencional e com solução salina, de sementes de cinco lotes de pepino, cultivar Rubi. Natal, EMPARN, 2004.

\begin{tabular}{|c|c|c|c|c|c|c|}
\hline \multirow{2}{*}{ Lote } & \multicolumn{3}{|c|}{$38^{\circ} \mathrm{C}$} & \multicolumn{3}{|c|}{$41^{\circ} \mathrm{C}$} \\
\hline & $48 \mathrm{~h}$ & $72 \mathrm{~h}$ & $96 \mathrm{~h}$ & $48 \mathrm{~h}$ & $72 \mathrm{~h}$ & $96 \mathrm{~h}$ \\
\hline & \multicolumn{6}{|c|}{ Envelhecimento acelerado convencional } \\
\hline 1 & $88 \mathrm{~b}$ & $86 \mathrm{c}$ & $80 \mathrm{c}$ & $80 \mathrm{~b}$ & $78 \mathrm{c}$ & $70 \mathrm{c}$ \\
\hline 2 & 96 a & 96 a & 94 a & 96 a & 94 a & $90 \mathrm{a}$ \\
\hline 3 & 95 a & $90 \mathrm{ab}$ & 94 a & 95 a & 94 a & 92 a \\
\hline 4 & 97 a & 98 a & 96 a & 96 a & 96 a & 94 a \\
\hline 5 & 96 a & $96 \mathrm{a}$ & $86 \mathrm{~b}$ & $88 \mathrm{~b}$ & $84 \mathrm{~b}$ & $80 \mathrm{~b}$ \\
\hline Média & $94 \mathrm{a}$ & $93 a b$ & $90 \mathrm{ab}$ & $91 \mathrm{ab}$ & $89 \mathrm{~b}$ & $85 a b$ \\
\hline \multicolumn{7}{|c|}{$\mathrm{CV}(\%)=5,2$} \\
\hline & \multicolumn{6}{|c|}{ Envelhecimento acelerado com solução salina } \\
\hline 1 & $88 \mathrm{~b}$ & $78 \mathrm{c}$ & $75 \mathrm{c}$ & $87 \mathrm{~b}$ & $78 \mathrm{c}$ & $72 \mathrm{c}$ \\
\hline 2 & 95 a & 96 a & 94 a & 96 a & 96 a & $94 \mathrm{ab}$ \\
\hline 3 & 96 a & 95 a & 94 a & $94 \mathrm{ab}$ & $92 \mathrm{ab}$ & $90 \mathrm{ab}$ \\
\hline 4 & 94 a & 96 a & 96 a & 96 a & 96 a & 96 a \\
\hline 5 & $92 \mathrm{a}$ & $88 \mathrm{~b}$ & $84 \mathrm{~b}$ & $92 \mathrm{ab}$ & $88 \mathrm{~b}$ & $87 \mathrm{~b}$ \\
\hline Média & 93 a & $91 \mathrm{~b}$ & $89 a b$ & $93 a b$ & $90 \mathrm{~b}$ & $88 \mathrm{~b}$ \\
\hline
\end{tabular}

"Na coluna, valores seguidos pela mesma letra não diferem entre si pelo teste de Tukey a 5\%.

perda de viabilidade da semente, caracterizada por ser relativamente longa e com poucas sementes mortas.

Os resultados do teste emergência de plântulas em casa de vegetação (Tabela 1) indicaram a menor qualidade do lote 1, diferindo estatisticamente dos lotes 2, 3, 4, e 5. Segundo Marcos-Filho (1999a), o teste de emergência de plântulas constitui parâmetro indicador da eficiência dos testes para avaliação do potencial fisiológico dos lotes de sementes. Portanto, verifica-se que essa eficiência em distinguir, com segurança, os lotes de baixo e alto vigor não foi tão eficiente na identificação de lotes com diferentes níveis de vigor, indicando apenas o lote 1 como o de menor potencial fisiológico. Assim, enfatizase a importância do uso de mais de um teste para determinar o vigor dos lotes de sementes (Marcos-Filho, 1998), devido à influência dos métodos adotados e uso de situações específicas de estresse para estimar o comportamento relativo dos lotes em campo (CARVALHO; NAKAGAWA, 2000).

De forma geral, ambos os períodos de envelhecimento (procedimento convencional) (Tabela 2 ), tanto a $38^{\circ} \mathrm{C}$ como a $41^{\circ} \mathrm{C}$, proporcionaram a identificação do lote 1 como o de menor potencial fisiológico, concordando com os resultados do teste de emergência de plântulas.
Nesse sentido, verifica-se que as combinações $38^{\circ} \mathrm{C} / 72$ e $96 \mathrm{~h}$ e $41^{\circ} \mathrm{C} / 72$ e $96 \mathrm{~h}$ foram as mais eficientes na identificação de diferenças entre os lotes avaliados. Estes resultados concordam parcialmente com os obtidos por Bhéring et al. (2000), que, trabalhando com sementes de pepino, recomendaram o período de $48 \mathrm{~h} \mathrm{a} 41^{\circ} \mathrm{C}$.

Apesar da semelhança entre as informações proporcionadas pelos períodos de envelhecimento convencional (Tabela 2) e os resultados de emergência de plântulas (Tabela 1), indicando o lote de menor qualidade, o período de $48 \mathrm{~h}$ de envelhecimento, para ambas as temperaturas, mostrou-se menos eficiente na separação dos lotes em diferentes níveis de vigor. Utilizando este mesmo período, a $42^{\circ} \mathrm{C}$, para avaliação de sementes de melão, Cano-Ríos et al. (2000), também não obtiveram resultados satisfatórios.

De um modo geral, a temperatura de $41^{\circ} \mathrm{C}$ promoveu maior redução sobre a germinação que a de $38^{\circ} \mathrm{C}$, principalmente para os lotes considerados de menor potencial fisiológico; a duração dos períodos de envelhecimento se mostrou menos severa que a elevação da temperatura. Esse fato concorda com as observações de Tomes et al. (1988), que sugeriram atenção especial ao monitoramento da temperatura durante o teste.
Para ambas as temperaturas, os períodos de 72 e 96h exibiram maior sensibilidade para identificação dos lotes em diferentes níveis de potencial fisiológico. Assim, os lotes 2, 3 e 4 foram os de melhor potencial fisiológico; o lote 1 mostrou pior qualidade e o lote 5 qualidade intermediária. Esses resultados concordam com os obtidos para o teste de emergência de plântulas em casa de vegetação, ao indicar a inferioridade do lote 1 (Tabela 1).

Portanto, as combinações $38^{\circ} \mathrm{C} / 72$ e $96 \mathrm{~h}$ e $41^{\circ} \mathrm{C} / 72$ e $96 \mathrm{~h}$ foram as mais eficientes na identificação de diferenças entre os lotes avaliados. Estes resultados estão, de certa forma, em conformidade com os encontrados por alguns autores para sementes de curcubitáceas, envolvendo diferentes períodos e temperatura, entre os quais destacam-se o de sementes de melancia, $45^{\circ} \mathrm{C} / 144 \mathrm{~h}$ (DELOUCHE; BASKIN, 1973), $41^{\circ} \mathrm{C} /$ 48 ou 72h (TRIGO; TRIGO, 1995).

O grau de umidade das sementes, após os períodos de envelhecimento convencional (Tabela 3 ), variou entre os lotes de $0,5 \%$ a $2,1 \%$, dependendo da combinação período/temperatura utilizada. Esta variação relativamente pequena no grau de umidade das sementes vai de encontro aos resultados encontrados por Panobianco e Marcos-Filho (1998), para sementes de pimentão, onde constataram elevação acentuada no grau de umidade após o envelhecimento acelerado, atingindo valores entre 29,5 e $37,8 \%$.

É conveniente a comparação de amostras que apresentem graus de umidade semelhantes antes do envelhecimento, embora diferenças de 1 a $2 \%$ não sejam comprometedoras (MARCOS-FILHO, 1999b). Para este estudo, o grau de umidade das sementes foi praticamente o mesmo (Tabela 1). Por outro lado, um dos principais indicadores da uniformidade das condições do envelhecimento acelerado é o grau de umidade das sementes ao final do teste, pois variações de 3 a $4 \%$ entre amostras são consideradas toleráveis (MARCOS-FILHO, 1999b); verificou-se que a variação máxima encontrada foi de $3,3 \%$, ou seja, dentro dos limites toleráveis (Tabela 3), assegurando a consistência das informações obtidas.

$\mathrm{O}$ teste de envelhecimento acelerado com solução salina (Tabela 2), o período de 48 horas, em ambas as temperaturas, revelou, de modo geral, resul- 
tados semelhantes aos obtidos para o envelhecimento convencional, apesar da menor eficiência na separação dos lotes. Houve, portanto, concordância com o teste de emergência de plântulas (Tabela 1), em termos de identificação dos lotes de menor potencial fisiológico. Os períodos de 72 e 96h, em ambas as temperaturas, apresentaram classificação mais evidente dos lotes em diferentes níveis de vigor, apontando os lotes 2, 3 e 4 como mais vigorosos e o lote 1 como o de pior qualidade. De forma geral, esses resultados estão compatíveis com os obtidos no teste de emergência de plântulas (Tabela 1) e envelhecimento convencional (Tabela 2).

Sendo assim, o teste de envelhecimento acelerado (procedimento convencional e com solução salina) conduzido com períodos de exposição de 72 e $96 \mathrm{~h}$ a 38 ou $41^{\circ} \mathrm{C}$ apresenta sensibilidade suficiente para avaliação do potencial fisiológico de sementes de pepino.

Com relação ao grau de umidade das sementes após os períodos de envelhecimento com solução salina (Tabela 3), os resultados foram semelhantes para os cinco lotes estudados. O grau de umidade das sementes expostas à solução saturada de $\mathrm{NaCl}$ apresentou valores menores e mais uniformes, após os períodos de envelhecimento em relação aos observados para as envelhecidas pelo procedimento convencional (Tabela 3); isto indica que o uso de solução salina contribui para retardar a absorção de água pelas sementes no teste de envelhecimento acelerado. Autilização de soluções saturadas de sais contribuiu também para reduzir acentuadamente ou impedir o desenvolvimento de microrganismos, minimizando, assim, a interferência de fator adicional nos resultados do teste (JIANHUA; MCDONALD, 1997).

Diante do exposto, verificam-se que as informações obtidas poderão auxiliar na tomada de decisões em diferentes etapas da produção e do uso de sementes, incluindo os trabalhos de seleção de lotes para semeadura, avaliação do potencial armazenamento, uso nos programas de controle de qualidade e também auxiliando nos métodos de seleção no melhoramento de plantas.

Portanto, neste estudo verificou-se que o teste de envelhecimento acelerado (procedimento convencional e com solução salina), conduzido com períodos de exposição de 72 e 96 h a 38 ou $41^{\circ} \mathrm{C}$, demonstrou sensibilidade suficiente para a avaliação do potencial fi-

Tabela 3. Graus de umidade (\%) obtidos após os testes de envelhecimento acelerado convencional e com solução salina de cinco lotes de sementes de pepino, cultivar Rubi. Natal, EMPARN, 2004.

\begin{tabular}{|c|c|c|c|c|c|c|}
\hline \multirow{2}{*}{ Lote } & \multicolumn{3}{|c|}{$38^{\circ} \mathrm{C}$} & \multicolumn{3}{|c|}{$41^{\circ} \mathrm{C}$} \\
\hline & $48 \mathrm{~h}$ & $72 \mathrm{~h}$ & $96 \mathrm{~h}$ & $48 \mathrm{~h}$ & $72 \mathrm{~h}$ & $96 \mathrm{~h}$ \\
\hline & \multicolumn{6}{|c|}{ Envelhecimento acelerado (convencional) } \\
\hline 1 & 27,0 & 29,0 & 29,5 & 26,4 & 27,6 & 28,0 \\
\hline 2 & 26,5 & 29,2 & 29,4 & 25,9 & 29,0 & 28,5 \\
\hline 3 & 26,9 & 29,7 & 31,0 & 25,7 & 28,0 & 28,4 \\
\hline 4 & 25,9 & 30,2 & 30,5 & 26,9 & 28,3 & 28,3 \\
\hline \multirow[t]{2}{*}{5} & 26,0 & 29,0 & 28,9 & 26,0 & 27,0 & 28,0 \\
\hline & \multicolumn{6}{|c|}{ Envelhecimento acelerado com solução salina } \\
\hline 1 & 9,8 & 10,3 & 10,2 & 8,6 & 9,4 & 9,2 \\
\hline 2 & 9,6 & 9,9 & 10,2 & 8,6 & 9,0 & 9,1 \\
\hline 3 & 9,5 & 10,5 & 9,9 & 7,9 & 9,3 & 9,9 \\
\hline 4 & 9,9 & 10,4 & 9,8 & 9,0 & 9,5 & 9,6 \\
\hline 5 & 9,7 & 10,0 & 10,3 & 9,0 & 9,4 & 9,6 \\
\hline
\end{tabular}

siológico de sementes de pepino. Verificou-se também que a utilização de solução saturada de $\mathrm{NaCl}$ contribui para reduzir a absorção de água pelas sementes de pepino durante o teste de envelhecimento acelerado, acarretou taxa de deterioração menos acentuada, resultados menos drásticos e mais uniformes, sem afetar a eficiência do teste.

\section{LITERATURA CITADA}

AGRIANUAL 2004. Anuário da agricultura brasileira. São Paulo: Editora Argos Comunicação, 2003. 496 p.

BENNETT, M.A.; EVANS, A.F.; GRASSBAUGH, E.M. Saturated salt accelerated aging (SSAA) test for assessing and comparing sh2 and se sweet corn seed lots. In: CONGRESS OF ISTA, 26., Angers, 2001. Abstracts appendix. Angers: ISTA, 2001. p.11.

BHÉRING, M.C.; DIAS, D.C.F.S.; GOMES, J.M.; BARROS, D.I. Métodos para avaliação do vigor de sementes de pepino. Revista Brasileira de Sementes, v.22, n.2, p.171-175, 2000.

BRASIL. Ministério da Agricultura e Reforma Agrária. Regras para análise de sementes. Brasília: SNDA; DNDV; CLAV, 1992. 365 p.

CARVALHO, N.M; NAKAGAWA, J. Sementes: ciencia, tecnologia e produção. 4 ed. Jaboticabal: FUNEP, 2000. 588 p.

CANO-RÍOS, P.; RAMÍREZ-ROSALES, G.; ORTEGÓN-PÉREZ, J.; ESPARZA-MARTÍNEZ, J.H.; RODRÍGUEZ-HERRERA, S. Análisis dialélico para vigor de semilla de melón. Agrociência, v.34, n.3, p.337-342, 2000.

DELOUCHE, J.C.; BASKIN, C.C. Accelerated aging techniques for predicting the relative storability of seed lots. Seed Science and Technology, v.1, n.2, p.427-452, 1973.

JIANHUA, Z:; McDONALD, M.B. The saturated salt accelerated aging test for small-seeded crops. Seed Science and Technology, v.25, n.1, p.123-131, 1997. MARCOS FILHO, J. O valor dos testes de vigor. Seed News, n.6, p.32, 1998.
MARCOS FILHO, J. Testes de vigor: importância e utilização. In: KRZYZANOWSKI, F.C.; VIEIRA, R.D.; FRANÇA-NETO, J.B. (Eds.).Vigor de sementes: conceitos e testes. Londrina: ABRATES, 1999a. cap.1, p.1-21.

MARCOS FILHO, J. Teste de envelhecimento acelerado. In: KRZYZANOWSKI, F.C.; VIEIRA, R.D.; FRANÇA NETO, J.B. (Ed.) Vigor de sementes: conceitos e testes. Londrina: ABRATES, 1999b. cap.3, p.1-24.

PANOBIANCO, M.; MARCOS-FILHO, J. Comparação entre métodos para avaliação da qualidade fisiológica de sementes de pimentão. Revista Brasileira de Sementes, v.20, n.2, p.306-310, 1998. PANOBIANCO, M.; MARCOS-FILHO, J. Envelhecimento acelerado e deterioração controlada em sementes de tomate. Scientia Agrícola, v.58, n.3, p.525-531, 2001.

POWELL, A.A.; MATTHEWS, S. Evaluation of controlled deterioration a new vigour test for small seeds vegetables. Seed Science and Technology, v.9, n.2, p.633-640, 1981.

POWELL, A.A. The controlled deterioration test. In: VAN DER VENTER, H.A. (Ed.) Seed vigour testing seminar. Copenhagen: The International Seed Testing Association, 1995. p.73-87.

RODO, A.B.; PANOBIANCO, M.; MARCOS-FILHO, J. Metodologia alternativa do teste de envelhecimento acelerado para sementes de cenoura. Scientia Agricola, v.57, n. 2, p.289-292, 2000.

TEBALDI, N.D.; SADER, R.; BIRUEL, R.P.; SCALON, N.J.O.; BALLARIS, A.L.; GAVIOLI, E. Determinação do tempo e da temperatura para o teste de envelhecimento acelerado de sementes de brócolos. (Brassica oleracea L.) var. italica Plenk. In: CONGRESSO BRASILEIRO DE SEMENTES, 11., Foz do Iguaçu, 1999. Resumos. Curitiba: ABRATES, 1999. p.120.

TEKRONY, D.M. Accelerated aging. In: VAN DE VENTER, H.A. (Ed.) Seed vigour testing seminar. Copenhagen: ISTA, 1995. p.53-72.

TOMES, L.T.; TEKRONY, D.M.; EGLI, D.B. Factors influencing the tray accelerated aging test for soybean seed. Journal of Seed Technology, v.12, n.1, p.24-36, 1988

TORRES, S.B.; MARCOS-FILHO, J. Accelerated ageing of melon seeds. Scientia Agricola, v.60, n.1, p.77-82, 2003.

TRIGO, M.F.O.O.; TRIGO, L.F.N. Avaliação do vigor em sementes de pepino (Cucumis sativus L.). Informativo ABRATES, Londrina, v.5, n.2, p.131, 1995. 